COMUNICACIÓN CIENTÍfICA IX CONGRESO NACIONAL DE LA SOCIEDAD CIENTÍFICA ESPAÑOLA DE ENFERMERÍA - SCELE. Mayo de 2018. UNIVERSIDAD DE ALICANTE.

\title{
SEGURIDAD EN EL USO DE MEDICAMENTOS EN ATENCIÓN PRIMARIA
}

L. Samper Soto

N. Hernández Ruiz

J. González Pastor

Hospital Universitario del Vinalopó. Elche. 


\section{PALABRAS CLAVE:}

SEGURIDAD, PACIENTE, MEDICAMENTO, PRIMARIA, FÁRMACO

\section{OBJETIVOS:}

Los Centros de Salud del Departamento 24, pertenecientes al Hospital Universitario del Vinalopó iniciaron su andadura en instaurar una cultura de seguridad del paciente en el año 2015 con el objetivo de conseguir la prestigiosa acreditación sanitaria Joint Commission International (JCI) que garantiza al paciente el cumplimiento de altos estándares de calidad y seguridad. Según el estudio APEAS un 48,2 \% de los eventos adversos están relacionados con la medicación en Atención Primaria, por ello la importancia de incorporar una cultura de seguridad en el uso de medicamentos entre nuestros profesionales sanitarios.

\section{MATERIAL Y METODOS:}

Para mejorar la seguridad en el uso de medicamentos nos hemos basado en siete elementos fundamentales:

- Identificación de los medicamentos de alto riesgo (MAR) en su ubicación con una pegatina roja (M.A.R)

- Identificación de los medicamentos de apariencia similar en su ubicación con una pegatina amarilla (PARECIDOS)

- Los electrolitos concentrados están correctamente identificados y almacenados únicamente en los almacenes autorizados.

- Los viales multidosis, una vez abiertos, disponen de fecha de apertura y caducidad.

- Aplicación de la regla de los cinco correctos antes de administrar una medicación.

- Registro adecuado en la historia clínica del paciente la administración de cada dosis de medicamento una vez administrado. Si no se administra registro del motivo.

- Si hay almacenamiento de un medicamento, comprobación de las condiciones especiales de conservación.

Se instaura la cultura de comunicación efectiva evitando órdenes verbales, presenciales o telefónicas, para la prescripción y administración de fármacos salvo en casos de emergencia clínica que se aplica el protocolo de actuación basado en la comunicación redundante.

Además de la creación de un circuito específico para la custodia, registro y uso de estupefacientes incorporando a un tercer testigo.

\section{RESULTADOS:}

Hemos consolidado en todos los profesionales de atención primaria una cultura de seguridad del paciente siendo el primer centro acreditado la zona básica de Crevillent por la organización con más experiencia en el sector sanitario, Joint Commision Internacional (JCI) en 2017 y actualmente en proceso de acreditar el resto de centros de nuestro departamento tras someternos a importantes auditorías donde se valoran 112 estándares que incluyen 455 elementos medibles relacionados con 5 grandes secciones como son, participación e integración en la comunidad, servicios centrados en el paciente, gestión de los servicios, mejora de la calidad y seguridad y objetivos internacionales de seguridad del paciente. Gracias a ello, hemos conseguido Implantar planes de mejora, creando una cultura de seguridad entre nuestros profesionales sanitarios y ofrecemos a nuestra población una calidad asistencial de alto nivel.

\section{DISCUSIÓN:}

Consideramos por tanto, la importancia de una cultura de seguridad en el entorno de Atención Primaria, creando y llevando el cumplimiento de objetivos/estándares para evitar el máximo de eventos adversos relacionados con el uso medicamentos. 